\title{
Testing Rebalancing Strategies for Stock-Bond Portfolios: What Is the Optimal Rebalancing Strategy?
}

\author{
Hubert Dichtl ${ }^{\mathrm{a}}$, Wolfgang Drobetz ${ }^{\mathrm{b}}$, and Martin Wambach ${ }^{\mathrm{c}}$ \\ This draft: January 2013
}

\begin{abstract}
We compare the performance of different rebalancing strategies under realistic market conditions by reporting statistical significance levels. Our analysis is based on historical data from the United States, the United Kingdom, as well as Germany and comprises three different classes of rebalancing (namely periodic, threshold, and range rebalancing). Despite cross-country differences, we provide evidence that both excessive as well as too infrequent rebalancing lead to an inferior risk-adjusted portfolio performance. Specifically, the optimal rebalancing strategy seems to be quarterly periodic rebalancing for all three countries under investigation.
\end{abstract}

\footnotetext{
${ }^{a}$ Hubert Dichtl, alpha portfolio advisors GmbH, Wiesbadener Weg 2a, 65812 Bad Soden/Ts., Germany, Mail: dichtl@ alphaport.de.

${ }^{\mathrm{b}}$ Wolfgang Drobetz, Institute of Finance, University of Hamburg, Von-Melle-Park 5, 20146 Hamburg, Germany, Mail: wolfgang.drobetz@wiso.uni-hamburg.de.

c Martin Wambach, Institute of Finance, University of Hamburg, Von-Melle-Park 5, 20146 Hamburg, Germany, Mail: martin.wambach@wiso.uni-hamburg.de.
} 
Once an investor's return and risk preferences as well as his regulatory environment have been identified, it is the primary objective of any institutional asset manager to implement and supervise the most suitable asset allocation for his client. Given this initial asset allocation, the literature differentiates between three reasons for portfolio rebalancing: (i) rebalancing due to a shift in an investor's risk profile and/or modified regulatory requirements; (ii) rebalancing based on changes in the expectations of future returns and risks; and (iii) rebalancing due to market movements. As discussed in Fabozzi, Focardi, and Kolm (2006) as well as in Leibowitz and Bova (2011), the first two reasons require the asset manager to construct a new optimal portfolio.

In this study, we focus on the third reason, which legitimates portfolio rebalancing as a cost-efficient, rule-based investment strategy. As different assets generate different rates of return, a portfolio’s relative asset composition will deviate from the target weights over time. In order to remain consistent with the institutional investor's initially evaluated return and risk preferences, the portfolio manager needs to rebalance the assets back to their predefined target weights. The resulting research question is which rebalancing algorithm and which rebalancing frequency should be adopted. In short, what is the optimal rebalancing strategy?

This issue is of considerable importance for investment practice as exemplarily documented by the Norwegian Government Pension Fund Global (NGPFG). Being one of the world's largest institutional investors with 554.96 billion US\$ AuM by the end of December 2011, the NGPFG is a good example of pursuing rebalancing as a cost-efficient, rule-based investment strategy (Norwegian Ministry of Finance, 2012). In search of the optimal risk-return reward, even small deviations from the strategic asset allocation could be economically relevant. In particular, this applies to very large funds which are restricted by their investment decisions according to their size. With 19.34 billion US\$ AuM by the end of June 2012, the Yale endowment also conducts rebalancing with the primary goal of maintaining the original 
risk profile as well as exploiting return-generating opportunities caused by excess security price volatility (Swensen, 2009; The Yale Endowment, 2012).

Examining dynamic portfolio strategies that invest only in stocks and bills, Perold and Sharpe (1988) have laid the theoretical foundations for our empirical analysis. They point out that the upside potential of a buy-and-hold strategy is proportional to the amount allocated into stocks, while its downside protection is proportional to the amount allocated into bills. Analyzing rebalancing strategies, three important conclusions can be drawn from the regular reallocation to the weaker performing asset class. First of all, rebalancing exhibits a lower upside potential in comparison to buy-and-hold during a persistent market upswing. Secondly, representing the sale of portfolio insurance, rebalancing also provides less downside protection in persistent market downswings. Nevertheless, rebalancing performs best in volatile markets that feature neither a persistent market downswing nor a persistent market upswing. According to Perold and Sharpe (1988), these market conditions advantage rebalancing strategies, which may ultimately result in both improved portfolio returns and a reduction of portfolio risk.

Investigating the average return, the volatility, and the Treynor ratio of several rebalancing strategies over the period from 1968 to 1991, Arnott and Lovell (1993) document that a monthly rebalancing strategy features the highest return while the corresponding volatility is only slightly higher compared to the strategy with the lowest volatility. However, using the Treynor ratio as a performance measure that incorporates both a strategy's return and its systematic risk, the empirical results are weaker. In fact, during the underlying 24-year sample period, all Treynor ratios lie very close together within the interval [0.784; 0.794], and thus it is not obvious which strategy actually performs best. Nevertheless, inferring from their analysis that rebalancing offers enhanced returns without increasing risk, Arnott and Lovell (1993) recommend a monthly rebalancing strategy to investors with a long investment horizon. 
Evaluating the performance on the basis of the Sharpe ratio over the period from 1986 to 2000 for different risk-profiles, Tsai (2001) shows that a frequent reallocation back to the target weights seems to provide some value added to institutional investors. ${ }^{1}$ However, as no single strategy is consistently better across portfolios of different risk profiles, Tsai (2001) argues that it does not matter much which rebalancing strategy is adopted.

Examining the period from 1995 to 2004, Harjoto and Jones (2006) report that a rebalancing strategy with an incorporated no-trade interval of $15 \%$ leads to both the highest average return and the lowest standard deviation, which in turn also results in the highest Sharpe ratio. This empirical finding also remains valid when the sample period is divided into an economic boom, a bust, and a recovery subsample. Taken as a whole, Harjoto and Jones (2006) conclude that investors should readjust their portfolio structure, though not too frequently. Nevertheless, three potential drawbacks are worth noting: (i) The analysis is based on one single 10-year period, which intensifies the potential problem of data snooping; (ii) transaction costs should have been incorporated because they might have a major influence on any reallocation decisions; (iii) the bust and recovery periods may not represent suitable estimators as they are based on only 27 and 30 observations, respectively.

Analyzing several rebalancing strategies over the period from 1926 to 2009, Jaconetti, Kinniry, and Zilbering (2010) show that buy-and-hold exhibits the highest average annualized return with a value of $9.1 \%$ after an investment period of 84 years, but also the highest volatility with a value of $14.4 \%$ due to an average stock allocation of $84.1 \%$. All remaining rebalancing strategies feature average returns that differ only slightly, ranging between $8.5 \%$ and $8.8 \%$. The standard deviations also lie within a narrow band of $11.8 \%$ to $12.3 \%$. While it is evident that most institutional investors cannot apply a buy-and-hold strategy on a long-

1 Tsai (2001) constructs five stock-bond portfolios with a 20\%, 40\%, 60\%, $80 \%$, and $98 \%$ equity allocation, respectively. The varying portfolio compositions are assumed to represent different risk profiles of institutional investors. Transaction costs are omitted, which weakens the explanatory power of her results. 
term basis, it is again not obvious which rebalancing strategy leads to superior results. Accordingly, Jaconetti, Kinniry, and Zilbering (2010) conclude that there is no universally optimal rebalancing strategy.

The mixed results of the studies presented above can be explained by the path-dependency of rebalancing which affects all dynamic portfolio strategies. As capital markets do not exhibit arbitrage opportunities over prolonged periods of time, this path-dependency further implies that there is no particular rebalancing strategy that features a better risk-return reward in all market environments in comparison to any other rebalancing strategy. However, the question that remains to be answered is whether a specific rebalancing strategy leads to a higher risk-adjusted performance on average.

Therefore, our first contribution to the literature relates to the implemented methodological approach, which is based on the stationary bootstrap of Politis and Romano (1994) and enables us to compare different rebalancing strategies with each other on a statistical basis by reporting statistical significance levels. Secondly, academic literature has so far remained incomplete by having excluded analyses of rebalancing strategies with a focus on institutional investors outside the US. For this reason, we do not only examine the financial markets of the United States, but also those of the United Kingdom and Germany in order to check whether our results are robust across countries or whether country-specific characteristics have an impact on the performance of rebalancing.

Our findings have immediate practical implications. First of all, evaluating risk-adjusted portfolio performance on the basis of the Sharpe ratio, the Sortino ratio, and the Omega measure, we provide evidence that both excessive rebalancing (monthly periodic rebalancing) as well as too infrequent rebalancing (yearly range rebalancing) provoke inferior results, thus pointing out that there may be an optimal rebalancing strategy. Secondly, the optimal trading patterns change with respect to the underlying rebalancing algorithm. Within the correspond- 
ing rebalancing class, quarterly periodic, quarterly threshold, and monthly range rebalancing seem to produce the highest risk-adjusted performance. Thirdly, quarterly periodic rebalancing significantly outperforms quarterly threshold rebalancing as well as monthly range rebalancing for all countries and all investment horizons. Overall, our results provide strong evidence that quarterly periodic rebalancing tends to be the optimal rebalancing strategy for all three countries under investigation. Fourthly, short-term momentum seems to be the primary source capable of explaining the statistically significant differences between monthly and quarterly periodic rebalancing.

The remainder of this paper proceeds as follows: Section I provides an overview of the implemented rebalancing strategies. Section II presents our methodology. We discuss the empirical results in Section III and conclude the paper in Section IV by drawing recommendations for the investment practice.

\section{Implemented Rebalancing Strategies}

Two different types of rebalancing have to be distinguished in the investment practice: periodic and interval rebalancing. While periodic rebalancing demands a reallocation to the predetermined target weights at the end of each period, interval rebalancing requires the implementation of a no-trade region around those target weights. In this study, we concentrate on a combination of both strategies: periodic rebalancing with the additional option of incorporating a symmetric no-trade region around the target weights.

\section{[Insert Table I here]}

Table I presents a classification of all rebalancing strategies under investigation: periodic, threshold, and range rebalancing with yearly, quarterly, and monthly trading intervals. In case of interval rebalancing, the portfolio manager must further differentiate between a reallocation to the target weights (threshold rebalancing) and to the nearest edge of the target weights 
(range rebalancing). While it is evident that the implementation of a no-trade region reduces transaction costs as an immediate consequence of a reduced portfolio turnover, our analysis in Section III sheds light on whether the additional utility of the reduced transaction costs will exceed the utility of a modified risk-return profile. In order to make our empirical results comparable with the investment practice, we mimic the long-term strategy of the NGPFG by implementing a symmetric no-trade region of $\pm 3 \%$ around the target weights (Norwegian Ministry of Finance, 2012)2.

We explain the exact procedure of the two different classes of interval rebalancing with the help of an example. Assume an investor of a two-asset portfolio with a strategic asset allocation of $60 \%$ stocks and $40 \%$ government bonds. Further assume a quarterly rebalancing frequency and a no-trade region of $\pm 3 \%$ around the target weights. Conducting a ' $3 \%$ quarterly threshold rebalancing strategy' requires a rebalancing back to the target weights of $60 \%$ stocks whenever the relative market capitalization of stocks has moved outside the no-trade region of [57\%; 63\%] at the end of each quarter. Otherwise, no transactions take place. Supervising a ' $3 \%$ quarterly range rebalancing strategy', the portfolio manager again has to check at the end of each quarter whether the relative market capitalization of stocks has fallen under $57 \%$ or has risen above $63 \%$. However, in the first case, a rebalancing to the lower threshold of $57 \%$ is necessary, whereas in the second case the relative stock market capitalization has to be adjusted to the upper threshold of $63 \%$ by the portfolio manager. If the stocks relative market capitalization lies between [57\%; 63\%] at the end of the quarter, again no transactions will take place.

2 Basically, the long-term strategy of the NGPFG can be characterized by a quarterly trading frequency, an implemented no-trade region of $\pm 3 \%$ around the target weights, and a reallocation back to the target weights if the relative stock proportion of stocks has fallen outside the no-trade region for one day during the corresponding quarter (Norwegian Ministry of Finance, 2012). This strategy is comparable to the implemented ' $3 \%$ quarterly threshold rebalancing' (classified as strategy 5 in Table I). 


\section{Methodology}

\section{A. Data}

In order to conduct a statistical test, a sufficient number of observations is necessary. Ranging from January 1982 to December 2011, our sample period constitutes a reasonable trade-off between the availability of the time series and the number of countries to be included. Based on monthly return data of well-diversified stock and government bond market total return indices as well as money market rates from Thomson Datastream, our analysis comprises the financial markets of the United States, the United Kingdom, and Germany. Moreover, in order to appropriately reflect real-world practice, the maturities of the government bond time series, namely 5, 7, and 10 years, also determine the corresponding investment horizons to be analyzed. All featuring a maturity of 3 months, we apply Treasury bills (United States), LIBOR (United Kingdom), and FIBOR (Germany) as proxies for the risk-free rate.

[Insert Table II here]

Table II presents the descriptive statistics of our dataset. As shown in Panel A of Table II, there are substantial differences between the capital markets of the United States, the United Kingdom, and Germany during the underlying 30-year sample period. For example, the German stock market exhibits the lowest average return with a value of $8.75 \%$, while it also features the highest volatility with a value $22.06 \%$. In contrast, the average stock market return for the United Kingdom is $10.84 \%$ with a volatility of $16.14 \%$. Dividing the 30 -year sample period of the United States financial markets' into two disjunctive 15-year sub-periods, Panel B of Table II further illustrates that the time series characteristics themselves can change over time. In particular, this is obvious for the average returns of all three asset classes. The stock market return has decreased from $15.59 \%$ in the first 15 -year subsample to $5.31 \%$ in the sec- 
ond one, while the government bond market return has decreased from $10.66 \%$ to $6.48 \%$, and the cash market return from $6.21 \%$ to $2.71 \%$. As all these time series characteristics will have an impact on the performance of rebalancing, not only an analysis of each country is necessary, but also a methodological approach that allows to preserve most of the time series characteristics and financial market dependencies.

\section{B. Settings}

Evaluating the performance of different rebalancing strategies, we focus on a $60 \%$ stocks and $40 \%$ government bonds asset allocation for three different reasons. First of all, our analysis considers three different countries (United States, United Kingdom, and Germany), three different classes of rebalancing (periodic, threshold, and range rebalancing), three different investment horizons (5, 7, and 10 years), and three different risk-adjusted performance measures (Sharpe ratio, Sortino ratio, and Omega measure). As all these parameters are linked by multiplication, we thus fix the initial asset allocation at $60 \%$ stocks and $40 \%$ government bonds in order to concentrate on our primary research question. Secondly, representing one of the world's largest institutional investors by the end of 2011, the NGPFG is a predominant example of having pursued a $60 \%$ stocks and $40 \%$ government bonds asset allocation in the past, thereby reflecting the high relevance for investment practice (Chambers, Dimson, and Ilmanen, 2012; Norwegian Ministry of Finance, 2012). Thirdly, a 60/40 asset allocation also enables us to compare and discuss our empirical results with the findings of prior rebalancing studies.

Moreover, we incorporate realistic transaction costs quoted at 15 bps per roundtrip. In particular, applying well-diversified stock market as well as government bond total return indices, we quote 10 bps for buying/selling stocks and 5 bps for buying/selling bonds. 


\section{Motivation}

The primary objective of our analysis is the statistical comparison of the performance of different rebalancing strategies under realistic market conditions. For this reason, our simulation approach is based on historical data. The implementation of the stationary bootstrap of Politis and Romano (1994) further enables us to preserve time series characteristics and financial market dependencies (such as positive autocorrelation in the short-run, heteroskedasticity, fat tails, left-skewed return distributions, and asset class correlations) to the greatest possible extent and to derive valuable recommendations for portfolio management.

So far, Monte Carlo simulations have been a suitable approach to analyze the impact of different market conditions on the performance of rebalancing strategies (Jones and Stine, 2010; Sun et al., 2006; Donohue and Yip, 2003; and Buetow et al., 2002). However, the rising frequency of financial crises provides strong evidence that commonly used probability distribution functions, such as the normal distribution or the t-distribution, seem to be no longer appropriate for modeling financial markets. Not only the descriptive statistics of Table II substantiates this observation, but also Annaert, Van Osselaer, and Verstraete (2009), among many others, who document that financial return series tend to be left-skewed and exhibit fat tails as well as heteroskedasticity. Moreover, as illustrated by Panel B of Table II, time series characteristics must not be necessarily stable over time. For example, the studies of Ferson, Kandel, and Stambaugh $(1987)$ as well as Ferson and Harvey $(1991,1993)$ all contribute to the explanation of time-varying risk premia. Engle (1982), Engle, Lilien, and Robins (1987), and Dumas and Solnik (1995) provide analyses on time-varying risk, and Erb, Harvey, and Viskanta (1994), Ball and Torous (2000), Longin and Solnik (2001), and Buraschi, Porchia, and Trojani (2010) examine time-varying asset class correlations. In summary, all these findings indicate that an appropriate calibration of the parameters for a Monte Carlo simulation can be extremely difficult. 
Analyses based on real world data avoid most of these difficulties. However, rebalancing studies that conduct simple historical analyses suffer from the drawback of examining only a single realization or a fairly small number of realizations. For example, Jaconetti, Kinniry, and Zilbering (2010) analyze an 84-year sample period from 1926 to 2009, Harjoto and Jones (2006) a 10-year sample period from 1995 to 2004, and Tsai (2001) a 15-year sample period from 1986 to 2000. Overall, Jaconetti, Kinniry, and Zilbering (2010) as well as Tsai (2001) both argue that there is no universally optimal rebalancing strategy. While Tsai (2001) concludes that it does not matter much which strategy is adopted, Jaconetti, Kinniry, and Zilbering (2010) recommend a semiannual or annual 5\% threshold rebalancing strategy. Moreover, Harjoto and Jones (2006) report that a 15\% monthly threshold rebalancing strategy is superior compared to other rebalancing strategies during all market phases. This issue of mixed results is reinforced by the fact that the performance of rebalancing is highly path-dependent because it constitutes a dynamic portfolio strategy. Therefore, it cannot be ruled out that the empirical results are also driven to a large extent by distinctive features of the underlying sample period rather than by the rebalancing strategy under investigation. Brock, Lakonishok, and LeBaron (1992) report that this danger of data snooping can be severe, and thus the empirical findings of simple historical analyses do not allow reliable interpretations.

\section{Test Design}

Based on the limitations of both Monte Carlo simulations and historical analyses, we perform historical simulations. Representing a reasonable trade-off, this approach enables us not only to capture most of the time series information, but also to conduct a statistical test, thereby clearly separating our analysis of the performance of rebalancing strategies from both Monte Carlo simulations and historical analyses. In particular, we apply the stationary bootstrap of Politis and Romano (1994) and test whether the mean of a difference time series is equal to zero: 
(1)

$$
H_{0}: \Delta_{P M}=0 \quad \text { versus } \quad H_{1}: \Delta_{P M} \neq 0 \text {, }
$$

where $P M$ constitutes the risk-adjusted performance measure, which is either the Sharpe ratio, the Sortino ratio, or the Omega measure. The difference between the two performance measures is given by:

$$
\Delta_{P M}=P M_{A}-P M_{B},
$$

where A and B denote rebalancing strategies as classified in Table I. The arithmetic mean is an appropriate point estimator of (2):

$$
\widehat{\Delta}_{P M}=\widehat{P M}_{A}-\widehat{P M}_{B} \text {. }
$$

Before executing our tests, the parameters 'performance measure', 'rebalancing class', ‘trading frequency', 'country’, and 'investment horizon' have to be specified. In a first step, we compare different rebalancing strategies within a given rebalancing class. As classified in Table I, this could either be periodic rebalancing, threshold rebalancing, or range rebalancing. After having determined the risk-adjusted performance measure of interest, we end up with three comparisons for each rebalancing class, each country, and each investment horizon:

$$
\begin{array}{ll}
\text { Monthly rebalancing - quarterly rebalancing } & (\mathrm{M}-\mathrm{Q}) \\
\text { Monthly rebalancing - yearly rebalancing } & (\mathrm{M}-\mathrm{Y}) \\
\text { Quarterly rebalancing - yearly rebalancing } & (\mathrm{Q}-\mathrm{Y})
\end{array}
$$

Having identified the optimal rebalancing strategy within each rebalancing class, we compare the performance differences between these three rebalancing strategies in a second step:

$$
\begin{aligned}
& \text { Periodic rebalancing - Threshold rebalancing } \\
& \text { Periodic rebalancing - Range rebalancing } \\
& \text { Threshold rebalancing - Range rebalancing }
\end{aligned}
$$

We implement the stationary bootstrap of Politis and Romano (1994). Being applicable to stationary, weakly dependent data, the stationary bootstrap allows us to efficiently exploit time series information by simulating realistic market conditions. In order to generate return paths that could have been realized in the past by drawing blocks of different lengths, we have to ascertain the probability $P$ for resampling the return observations. Following a geo- 
metric distribution, we take advantage of the resulting inverse relationship between $P$ and the average block size, which is the expected reciprocal value of $P$. We determine the optimal average block size by using the automatic block-length selection for the dependent bootstrap of Politis and White (2004), and we further incorporate the corrections made by Patton et al. (2009). Taken as a whole, an average block length of 2 is recommended for all stock and government bond time series of all three countries under investigation. Although Table II reports substantial cross-country differences, this finding allows us to compare our empirical results derived from the different financial markets of the United States, the United Kingdom, and Germany.

We start our analysis by bootstrapping pairwise 100 return paths of stocks, government bonds, and risk-free rates for each country under investigation. The pairwise resampling is necessary in order to preserve the cross-sectional dependency structure between stocks, government bonds, and risk-free-rates. The investment horizons to be analyzed, namely 5, 7, and 10 years, determine the length of the resampled return paths. In contrast to Ledoit and Wolf (2008, 2011) who examine a 10-year investment horizon by bootstrapping from a 10-year sample period, we resample investment horizons of 5, 7, and 10 years by drawing blocks of different lengths from the underlying 30-year sample period. This procedure enables us both to exploit the full information of the underlying sample period and to compare the impact of different investment horizons on the performance of rebalancing. In order to conduct statistical comparisons according to (4.1)-(4.6), we ascertain the rebalancing class, the trading frequency, and the performance measure of interest and calculate the mean for the corresponding difference time series. In a second step, we repeat this procedure B times in order to construct two-sided percentile intervals according to Efron and Tibshirani (1998):

$$
\hat{\Delta}_{P M[1]}^{*} \leq \hat{\Delta}_{P M[2]}^{*} \leq \ldots \leq \hat{\Delta}_{P M[B-1]}^{*} \leq \hat{\Delta}_{P M[B]}^{*},
$$


where (5) states the ordered difference series of the performance measure of interest. In this context, Romano and Wolf (2006) document that the studentized block bootstrap leads to an improved coverage accuracy for small sample sizes in comparison to normal theory intervals as well as the basic bootstrap. In case of small to moderate sample sizes, Ledoit and Wolf (2008, 2011) also suggest a studentized time series bootstrap if p-values need to be calculated. Nevertheless, covering 30 years with 360 monthly return observations, our sample period can be considered as large, legitimating the construction of percentile intervals as described by Efron and Tibshirani (1998):

(6) $\quad C I=\left[\widehat{\Delta}_{P M\left[\frac{\alpha}{2} \cdot B\right]}^{*}, \hat{\Delta}_{P M\left[\frac{1-\alpha}{2} \cdot B\right]}^{*}\right]$.

The null hypothesis $H_{0}$ is rejected at the significance level $\alpha$ if $0 \notin C I$. The nominal levels of $\alpha$ to be considered are $0.01,0.05$, and 0.10 . We conduct $B=1,000$ simulations. Repeated simulations reveal that our results are stable in capturing the underlying patterns in our sample.

\section{Empirical Simulation Results}

Taking both the return and the risk of a portfolio strategy into account, we apply the Sharpe ratio, the Sortino ratio, and the Omega measure in order to appropriately evaluate portfolio performance. We start our discussion by comparing the risk-adjusted performance on a statistical basis within each of the three rebalancing classes periodic, threshold, and range rebalancing. Our analysis proceeds with the statistical comparison of the risk-adjusted performance of rebalancing between these classes.

\section{A. Periodic Rebalancing}

The Sharpe ratio (Sharpe, 1966) is the most commonly used risk-adjusted performance measure in investment practice. Panel A of Table III shows the average annualized Sharpe 
ratios of periodic rebalancing classified by trading frequency, investment horizon, and country. On average, quarterly periodic rebalancing exhibits higher Sharpe ratios for all countries and all investment horizons under investigation compared to both monthly and yearly rebalancing. This finding provides a first hint that both too frequent as well as too infrequent rebalancing could lead to an inferior risk-adjusted portfolio performance.

\section{[Insert Table III here]}

Although we observe a similar pattern for all three countries under investigation, Table III also clearly illustrates the cross-country differences. Assuming a 10-year investment horizon and yearly periodic rebalancing by way of example, the average Sharpe ratio of the United States is 0.579, whereas the average Sharpe ratios of the United Kingdom and Germany are substantially lower with values of 0.389 and 0.355 , respectively. Again, classified by country and investment horizon, Table IV proves whether the differences in the average risk-adjusted performance reported in Table III are statistically significant or whether they can simply be ascribed to a distinctive feature of the underlying sample period. If both boundaries are positive (negative), the first rebalancing strategy causes a significantly higher (lower) average risk-adjusted performance compared to the second one. Otherwise, the confidence interval includes zero, implying that the difference is lost in estimation error and that no statistical inferences can be drawn. In eight out of nine cases, monthly periodic rebalancing leads to a significantly lower Sharpe ratio compared to quarterly periodic rebalancing. Although we cannot uncover statistical significance for the financial market of the United Kingdom with an underlying 5-year investment horizon, the position of the $10 \%$ quintile (indicated by the magnitude of the lower and upper boundary) suggests that quarterly periodic rebalancing seems to produce a superior risk-adjusted performance in terms of average Sharpe ratios in comparison to monthly periodic rebalancing as well. Even if - in most cases - no significance 
can be detected, the magnitude of the lower and the upper boundary of the underlying $10 \%$ quintiles in Panel A of Table IV further reveals that yearly periodic rebalancing also tends to produce inferior risk-adjusted average Sharpe ratios in comparison to quarterly periodic rebalancing.

\section{[Insert Table IV here]}

From an economic perspective, positive deviations from the target return are not expected to be perceived as risk by investors, but rather as an opportunity to generate an extra return on the invested capital. Therefore, in addition to the Sharpe ratio, we also apply the Sortino ratio, which only takes negative deviations from the expected return into account (Sortino and Price, 1994):

$$
S_{i}=\frac{\bar{r}_{i}-\tau}{\sqrt{\int_{-\infty}^{\tau}\left(\tau-r_{i}\right)^{2} f\left(r_{i}\right) d r_{i}}},
$$

where $\bar{r}_{i}$ is the average return of the underlying rebalancing strategy $i, f\left(r_{i}\right)$ the corresponding probability density function, and $\tau$ the target return required by the investor. We set the target return to zero, which allows us to differentiate between realized gains and losses. Although the economic impact seems to be small, Panel B of Table III substantiates the observation that there may be an optimal trading frequency. Again, quarterly periodic rebalancing produces the highest risk-adjusted portfolio performance for all countries and all investment horizons compared to both monthly and yearly periodic rebalancing. All in all, Panel B of Table IV reconfirms our findings in Panel A of Table IV. In five out of nine cases, quarterly periodic rebalancing leads to significantly higher average Sortino ratios compared to both monthly and yearly periodic rebalancing. With regard to the remaining cases, the positions of the $10 \%$ quintile also indicate without any exception that quarterly periodic rebalancing tends to exhibit a higher risk-adjusted portfolio performance in terms of average Sortino ratios. 
As neither the Sharpe ratio nor the Sortino ratio account for higher moments, such as the skewness of a return distribution or its kurtosis, portfolio recommendations derived on the basis of these risk-adjusted performance measures could be biased. By way of example, since the turn of the millennium, the dot.com bubble burst of 2000, the destabilization effects of 9/11, the subprime mortgage crisis of 2007, and - most recently - the European sovereign debt crisis of 2010 all have impressively shown that fat tails must not be ignored. For this reason, we additionally use the Omega measure, which considers the entire return distribution (Shadwick and Keating, 2002). Representing a special case of the more general performance measure Kappa (Kaplan and Knowles, 2004), it is defined as the ratio of gains to losses relative to a predefined target return:

$$
\Omega_{i}(\tau)=\frac{\int_{\tau}^{\infty}\left(1-F\left(r_{i}\right)\right) d r_{i}}{\int_{-\infty}^{\tau} F\left(r_{i}\right) d r_{i}}
$$

where $F\left(r_{i}\right)$ denotes the cumulative distribution function of the monthly return $r$ of rebalancing strategy $i$, and $\tau$ is the investor's required rate of return, which we again set to zero. The results in Panel C of Table III and in Panel C of Table IV are qualitatively similar to those in Panel B of Table III and in Panel B of Table IV, thereby substantiating the empirical finding that quarterly periodic rebalancing tends to exhibit a superior risk-adjusted performance compared to both monthly and yearly periodic rebalancing.

Taken as a whole, we conclude that both too frequent as well as too infrequent rebalancing results in a suboptimal portfolio performance. Following Ledoit and Wolf (2011), we also modify the average block length to six instead of two as a further robustness check. The results (not reported) are even stronger in this case. In fact, we even observe that monthly periodic rebalancing is significantly outperformed in comparison to quarterly periodic rebalancing for all three countries, all three investment horizons, and all three risk-adjusted performance measures under investigation. Therefore, our results contradict the recommendation of 
Arnott and Lovell (1993), who suggest that investors with a long investment horizon should rebalance on a monthly basis.

\section{B. Interval Rebalancing}

Searching for an optimal rebalancing strategy, we additionally test threshold and range rebalancing strategies by implementing a symmetric no-trade region around the target weights. Once a rebalancing threshold is introduced, there are two cases that need to be distinguished with regard to the practical implementation. If a rebalancing is necessary at the end of the predetermined period, the portfolio weights can be reallocated either to the original target weights (Buetow et al., 2002; Harjoto and Jones, 2006) or to the nearest edge of the original target weights (Leland, 1999). As explained in Section I, threshold rebalancing refers to strategies (4)-(6) whereas range rebalancing corresponds to strategies (7)-(9) in Table I.

Table V provides only weak statistical evidence that quarterly threshold rebalancing leads to a better risk-adjusted performance compared to both monthly and yearly threshold rebalancing. However, the positions of the $10 \%$ quintile again indicate that quarterly threshold rebalancing seems to produce the highest average risk-adjusted performance for all countries under investigation. This pattern changes with respect to range rebalancing. Table VI documents that yearly range rebalancing is significantly outperformed by both quarterly and monthly range rebalancing in almost all cases. Moreover, monthly range rebalancing also tends to feature a better risk-adjusted performance compared to quarterly range rebalancing.

\section{[Insert Table V and Table VI here]}

Overall, our results provide strong evidence that both excessive rebalancing (monthly periodic rebalancing) as well as too infrequent rebalancing (yearly range rebalancing) leads on average to inferior Sharpe ratios, Sortino ratios, and Omega measures. The related findings in Dichtl et al. (2012) substantiate our argumentation. In particular, they report that buy-and- 
hold - which implies no rebalancing at all - is outperformed on a risk-adjusted basis by all three rebalancing classes at the $1 \%$ level. As a consequence, our empirical findings indicate that there may be an optimal rebalancing strategy. However, this result contradicts the reasoning of Jaconetti, Kinniry, and Zilbering (2010) as well as that of Tsai (2001), who all conclude from their analyses that there is no universally optimal rebalancing strategy. In contrast, we show that the optimal trading patterns change with respect to the underlying rebalancing strategy (periodic, threshold or range rebalancing). While a quarterly trading frequency seems optimal for periodic and threshold rebalancing, it is a monthly trading frequency that tends to produce the best results for range rebalancing.

\section{Optimal Rebalancing}

Having analyzed the trading patterns within a given rebalancing class, the question of interest that now arises is which of these rebalancing strategies performs best. Therefore, we compare the average risk-adjusted performance of quarterly periodic rebalancing, quarterly threshold rebalancing, and monthly range rebalancing with each other. Table VII documents that monthly range rebalancing leads to a significantly lower risk-adjusted performance in terms of Sharpe ratios, Sortino ratios, and Omega measures at least at the $5 \%$ level compared with both quarterly periodic rebalancing and quarterly threshold rebalancing. Although we cannot detect statistical significance with regard to average Sharpe ratios, Panel B as well as Panel C of Table VII document that, on average, quarterly periodic rebalancing offers significantly higher Sortino ratios and Omega measures for all three countries and all investment horizons under investigation compared to quarterly threshold rebalancing. It is also noteworthy that statistical significance levels are much more pronounced for comparisons between different rebalancing classes in contrast to comparisons within a certain rebalancing class. Therefore, we conjecture that the rebalancing algorithm itself will have a higher impact on the performance of rebalancing compared to the trading frequency. 
In conclusion, if an investor identifies rebalancing as an appropriate portfolio strategy subject to the underlying return and risk preferences as well as the regulatory environment, our empirical simulation analysis supports a quarterly periodic rebalancing strategy. This finding is in line with the long-term strategy of the NGPFG, which also adopts a quarterly trading frequency (Chambers, Dimson, and Ilmanen, 2012; Norwegian Ministry of Finance, 2012). However, we further show that the benefit from reduced transaction costs due to the implemented no-trade region around the target weights does not outweigh a lower value added resulting from altered risk and return characteristics. With respect to the NGPFG, two additional arguments are worth noting that advantage quarterly periodic rebalancing in comparison to quarterly threshold rebalancing. First of all, transaction costs of the NGPFG are expected to be lower than 15 bps per roundtrip due to its bargaining power. Secondly, our analysis is based on lump-sum payments taking place at the beginning of the underlying investment horizon, whereas the NGPFG receives a steady cash inflow from selling a part of Norway's petroleum resources. Reducing the need for reallocating the portfolio weights, this partial rebalancing also contributes to saving transaction costs.

\section{[Insert Table VII here]}

Although the economic impact seems to be small at a first glance, it is an important finding for investment practice that our primary results are statistically significant and robust across countries. Even small differences are expected to be economically relevant if AuM are of considerable size. With over 550 billion US\$ AuM by the end of 2011, the NGPFG is a good example for a large institutional investor who conducts rebalancing as a cost-efficient rule-based investment strategy. In order to illustrate and compare the return potential between monthly and quarterly periodic rebalancing, we construct a hypothetical example similar to the NGPFG. Obtaining the input parameters from the financial market of the United States, 
we assume an average Sharpe ratio of 0.575 for monthly periodic rebalancing and 0.579 for quarterly periodic rebalancing, respectively. We further keep the underlying volatility constant at $10 \%$ p.a. ${ }^{3}$ The resulting implicit annual excess return would be $5.75 \%$ for monthly periodic rebalancing and 5.79\% for quarterly periodic rebalancing. Therefore, given that both strategies exhibit the same risk potential, the overall excess return potential would amount to 220 million US\$.

\section{Impact of Time Series Characteristics on Portfolio Performance}

A still open question is which driving force is responsible for the observation that, on average, monthly periodic rebalancing exhibits a lower risk-adjusted performance compared to quarterly periodic rebalancing. This result remains valid even if transaction costs are excluded from our analysis (not reported). Possible sources could be time series characteristics, such as short-term momentum and long-term mean-reversion, the cross-correlation between stocks and bonds, and distributional characteristics of the return generating process. In order to shed light on this issue, we conduct a simple Monte Carlo simulation, assuming a geometric Brownian motion with normally distributed stock and government bond markets returns as well as a correlation of zero in a first step. We further calibrate the parameters 'mean' and 'volatility' by applying the average values of the US financial markets over the entire 30-year sample period. Based on Table II, we use $10.45 \%$ (8.57\%) as the average annual sample mean for stocks (government bonds) and $15.77 \%$ (7.91\%) as the corresponding average annual volatility. Furthermore, we keep the risk-free rate constant at the long-term average of 4.46\%. Our simulations (results not tabulated) do not detect any differences in risk-adjusted performance measures between monthly and quarterly periodic rebalancing.

\footnotetext{
3 In results not shown, monthly periodic rebalancing exhibits - on average - a marginally lower annual return as well as a marginally higher annual risk in terms of volatility and semi-volatility for all countries and all investment horizons compared to quarterly periodic rebalancing, leading to inferior average Sharpe ratios.
} 
In a second step, we completely break down the time series structure of our real-world data by using a fixed block length of 1 in the stationary bootstrap. This procedure deletes shortterm momentum, but preserves both the distributional characteristics as well as the correlation structure between stocks and bonds. Again, we do not detect any statistical differences in risk-adjusted performance measures between monthly and quarterly periodic rebalancing (results not tabulated). As statistical significance completely disappears if the time series structure is destroyed, we conclude that time series characteristics - especially short-term momentum - are the primary sources capable of explaining the statistically significant differences in average Sharpe ratios between monthly and quarterly periodic rebalancing. While we do not have a simple explanation at hand, we suspect that the interrelations between returns, risk, and - in particular - portfolio weights are responsible for this finding.

\section{Concluding Remarks}

This study compares the risk-adjusted performance of different rebalancing strategies under realistic market conditions by reporting statistical significance levels. First of all, we document that monthly periodic rebalancing features a lower average risk-adjusted performance for all three countries and for all investment horizons under investigation in comparison to quarterly periodic rebalancing. Moreover, as yearly range rebalancing also leads to inferior Sharpe ratios, Sortino ratios, and Omega measures, our results imply that there is an optimal rebalancing strategy with both excessive rebalancing (monthly periodic rebalancing) as well as too infrequent rebalancing (yearly range rebalancing) provoking a suboptimal risk-adjusted performance. Secondly, the optimal trading frequency is subject to the underlying rebalancing algorithm. Within the corresponding rebalancing class, quarterly periodic, quarterly threshold, and monthly range rebalancing seem to produce the highest risk-adjusted performance. Thirdly, as quarterly periodic rebalancing leads to significantly higher average Sortino ratios and Omega ratios in comparison to quarterly threshold and monthly range re- 
balancing, our findings promote a quarterly periodic rebalancing strategy as it tends to produce the highest average risk-adjusted performance for all three countries under investigation. Lastly, short-term momentum seems to be the primary source capable of explaining the statistically significant differences between monthly and quarterly periodic rebalancing. 


\section{References}

Annaert, J., S. Van Osselaer, and B. Verstraete, 2009, "Performance Evaluation of Portfolio Insurance Strategies Using Stochastic Dominance Criteria,” Journal of Banking and Finance 33, 272-280.

Arnott, R.D. and R.M. Lovell, 1993, “Rebalancing: Why? When? How often?” Journal of Investing 2, 5-10.

Ball, C. and W.N. Torous, 2000, “Stochastic Correlation across International Stock Markets,” Journal of Empirical Finance 7, 373-388.

Brock, W., J. Lakonishok, and B. LeBaron, 1992, “Simple Technical Trading Rules and the Stochastic Properties of Stock Returns,” Journal of Finance 47, 1731-1764.

Buetow, G.W., E. Hunt, R. Sellers, D. Trotter, and W.A. Whipple, 2002, “The Benefits of Rebalancing,” Journal of Portfolio Management 28, 23-32.

Buraschi, A., P. Porchia, and F. Trojani, 2010, “Correlation Risk and Optimal Portfolio Choice,” Journal of Finance 65, 393-420.

Chambers, D., E. Dimson, and A. Ilmanen, 2012, “The Norway Model,” Journal of Portfolio Management 38, 67-81.

Dichtl, H., W. Drobetz, and M. Wambach, 2012, “Testing Rebalancing Strategies for StockBond Portfolios: Where Is the Value Added of Rebalancing?” Working Paper, University of Hamburg.

Donohue, C. and K. Yip, 2003, “Optimal Portfolio Rebalancing with Transaction Costs,” Journal of Portfolio Management 29, 49-63.

Dumas, B. and B. Solnik, 1995, “The World Price of Foreign Exchange Risk,” Journal of Finance 50, 445-479.

Efron, B. and R.J. Tibshirani, 1998, “An Introduction to the Bootstrap,” New York, Chapman \& Hall/CRC.

Engle, R.F., 1982, “Autoregressive Conditional Heteroscedasticity with Estimates of the Variance of United Kingdom Inflation,” Econometrica 50, 987-1007.

Engle, R.F., D.M. Lilien, and R.P. Robins, 1987, "Estimating Time-Varying Risk Premia in the Term Structure: The ARCH-M Model,” Econometrica 55, 391-408. 
Erb, C.B., C.R. Harvey, and T.E. Viskanta, 1994, “Forecasting International Equity Correlations,” Financial Analysts Journal 50, 32-45.

Fabozzi, F.J., S.M. Focardi, and P.N. Kolm, 2006, "Incorporating Trading Strategies in the Black-Litterman Framework,” Journal of Trading 1, 28-37.

Ferson, W.E. and C.R. Harvey, 1991, “The Variation of Economic Risk Premiums,” Journal of Political Economy 99, 385-415.

Ferson, W.E. and C.R. Harvey, 1993, "The Risk and Predictability of International Equity Returns,” Review of Financial Studies 6, 527-566.

Ferson, W.E., S. Kandel, and R.F. Stambaugh, 1987, “Tests of Asset Pricing with TimeVarying Expected Risk Premiums and Market Betas,” Journal of Finance 42, 201-220.

Harjoto, M.A. and F.J. Jones, 2006, "Rebalancing Strategies for Stocks and Bonds Asset Allocation,” Journal of Wealth Management 9, 37-44.

Jaconetti, C.M., F.M. Kinniry, and Y. Zilbering, 2010, "Best Practices for Portfolio Rebalancing," Vanguard, 1-17.

Jones, S.K. and J.B. Stine, 2010, “Expected Utility and the Non-Normal Returns of Common Portfolio Rebalancing Strategies,” Journal of Asset Management 10, 406-419.

Kaplan, P.D. and J.A. Knowles, 2004, “Kappa: A Generalized Downside Risk-Adjusted Performance Measure,” Journal of Performance Measurement 8, 42-54.

Ledoit, O. and M. Wolf, 2008, "Robust Performance Hypothesis Testing with the Sharpe Ratio,” Journal of Empirical Finance 15, 850-859.

Ledoit, O. and M. Wolf, 2011, “Robust Performance Hypothesis Testing with Variance,” Wilmott Magazine, 86-89.

Leibowitz, M. L. and A. Bova, 2011, “Policy Portfolios and Rebalancing Behavior,”. Journal of Portfolio Management 37, 60-71.

Leland, H. E., 1999, “Optimal Portfolio Management with Transactions Costs and Capital Gains Taxes,” Research Program in Finance Working paper RPF-290, University of California, Berkeley.

Longin, F. and B. Solnik, 2001, “Extreme Correlation of International Equity Markets,” Journal of Finance 56, 649-676. 
Norwegian Ministry of Finance, 2012, “The Management of the Government Pension Fund in 2011,” Report No. 17 to the Storting.

Patton, A., D.N. Politis, and H. White, 2009, “Correction to “Automatic Block-Length Selection for the Dependent Bootstrap” by D. Politis and H. White,” Econometric Reviews 28, 372-375.

Perold, A.F. and W.F. Sharpe, 1988, “Dynamic Strategies for Asset Allocation,” Financial Analysts Journal 44, 16-27.

Politis, D.N. and J.P. Romano, 1994, “The Stationary Bootstrap,” Journal of the American Statistical Association 89, 1303-1313.

Politis, D.N. and H. White, 2004, “Automatic Block-Length Selection for the Dependent Bootstrap,” Econometric Reviews 23, 53-70.

Romano, J.P. and M. Wolf, 2006, “Improved Nonparametric Confidence Intervals in Time Series Regressions,” Journal of Nonparametric Statistics 18, 199-214.

Sharpe, W.F., 1966, “Mutual Fund Performance,” Journal of Business 39, 119-138.

Shadwick, W.F. and C. Keating, 2002, “A Universal Performance Measure,” Journal of Performance Measurement 6, 59-84.

Sortino, F.A. and L.N. Price, 1994, "Performance Measurement in a Downside Risk Framework,” Journal of Investing 3, 59-64.

Swensen, D. F., 2009, "Pioneering Portfolio Management: An Unconventional Approach to Institutional Investment”, The Free Press, New York, NY.

Sun, W., A.C. Fan, L.-W. Chen, T. Schouwenaars, and M.A. Albota, 2006, “Optimal Rebalancing for Institutional Portfolios,” Journal of Portfolio Management 32, 33-43.

The Yale Endowment, 2012, “Endowment Update 2012”.

Tokat, Y. and N. Wicas, 2007, “Portfolio Rebalancing in Theory and Practice,” Journal of Investing 16, 52-59.

Tsai, C.S.-Y., 2001, “Rebalancing Diversified Portfolios of Various Risk Profiles,” Journal of Financial Planning 14, 104-110. 


\section{Table I. Classification of Implemented Rebalancing Strategies}

This table presents all rebalancing strategies under investigation. The periodic rebalancing strategies 1,2 , and 3 are characterized by a regular reallocation to the predetermined target weights at the end of each period. Strategies 4, 5, and 6 represent threshold rebalancing, which is classified as periodic interval rebalancing with a strict adjustment to the target weights. In contrast, the range rebalancing strategies 7,8 , and 9 require a reallocation to the nearest edge of the predefined interval boundaries. A threshold of $\pm 3 \%$ is applied to both threshold rebalancing and range rebalancing.

\begin{tabular}{lllllr}
\hline Rebalancing Strategy & Frequency & Threshold & Reallocation & Classification & No. \\
\hline Yearly Periodic Rebalancing & Yearly & No Threshold & Target Weights & Periodic & 1 \\
Quarterly Periodic Rebalancing & Quarterly & No Threshold & Target Weights & Periodic & 2 \\
Monthly Periodic Rebalancing & Monthly & No Threshold & Target Weights & Periodic & 3 \\
Yearly Threshold Rebalancing & Yearly & Threshold & Target Weights & Threshold & 4 \\
Quarterly Threshold Rebalancing & Quarterly & Threshold & Target Weights & Threshold & 5 \\
Monthly Threshold Rebalancing & Monthly & Threshold & Target Weights & Threshold & 6 \\
Yearly Range Rebalancing & Yearly & Threshold & Interval Boundaries & Range & 7 \\
Quarterly Range Rebalancing & Quarterly & Threshold & Interval Boundaries & Range & 8 \\
Monthly Range Rebalancing & Monthly & Threshold & Interval Boundaries & Range & 9 \\
\hline
\end{tabular}




\section{Table II. Descriptive Statistics}

Panel A presents the cross-sectional descriptive statistics of the stock, government bond, and money markets of the United States, the United Kingdom, and Germany over the entire 30-year sample period from January 1982 to December 2011. Panel B shows the descriptive statistics of the United States over the entire 30-year sample period as well as the two corresponding disjunctive 15-year subsamples. Bonds denote government bonds with a maturity of 10 years. Cash represents the corresponding 3-month money market rates. All statistics are calculated on a monthly basis using continuous compounded returns. Mean, Volatility, Skewness, and Kurtosis denote the annualized mean return, volatility, skewness, and kurtosis. Skewness and Kurtosis are calculated as the third and fourth normalized centered moments. Minimim and Maximum are the monthly minimum and maximum returns, respectively.

\begin{tabular}{|c|c|c|c|c|}
\hline \multicolumn{5}{|c|}{ Panel A: Cross-sectional descriptive statistics } \\
\hline Asset & Statistics & United States & United Kingdom & Germany \\
\hline \multirow[t]{6}{*}{ Stocks } & Mean (\%) & 10.45 & 10.84 & 8.75 \\
\hline & Volatility (\%) & 15.77 & 16.14 & 22.06 \\
\hline & Skewness & -0.91 & -1.15 & -0.92 \\
\hline & Kurtosis & 6.07 & 8.05 & 5.60 \\
\hline & Minimum (\%) & -23.85 & -30.02 & -28.67 \\
\hline & Maximum(\%) & 12.47 & 13.72 & 19.02 \\
\hline \multirow[t]{6}{*}{ Bonds } & Mean (\%) & 8.57 & 10.19 & 7.34 \\
\hline & Volatility (\%) & 7.91 & 8.01 & 5.53 \\
\hline & Skewness & 0.05 & -0.06 & -0.29 \\
\hline & Kurtosis & 3.66 & 4.45 & 3.26 \\
\hline & Minimum (\%) & -7.36 & -8.16 & -5.69 \\
\hline & Maximum (\%) & 9.40 & 8.17 & 5.37 \\
\hline \multirow[t]{6}{*}{ Cash (level) } & Mean (\%) & 4.46 & 6.91 & 4.43 \\
\hline & Volatility (\%) & 0.77 & 1.01 & 0.65 \\
\hline & Skewness & 0.16 & 0.23 & 0.55 \\
\hline & Kurtosis & 2.70 & 2.39 & 2.62 \\
\hline & Minimum(\%) & 0.00 & 0.00 & 0.00 \\
\hline & Maximum (\%) & 0.01 & 0.01 & 0.01 \\
\hline \multicolumn{5}{|c|}{ Panel B: Descriptive statistics of the United States for subsamples } \\
\hline & & Full sample & 1st half & 2nd half \\
\hline & & 年 & & \\
\hline \multirow[t]{6}{*}{ Stocks } & Mean (\%) & 10.45 & 15.59 & 5.31 \\
\hline & Volatility (\%) & 15.77 & 14.47 & 16.89 \\
\hline & Skewness & -0.91 & -1.12 & -0.71 \\
\hline & Kurtosis & 6.07 & 9.79 & 3.94 \\
\hline & Minimum(\%) & -23.85 & -23.85 & -18.76 \\
\hline & Maximum(\%) & 12.47 & 12.47 & 10.42 \\
\hline \multirow[t]{6}{*}{ Bonds } & Mean (\%) & 8.57 & 10.66 & 6.48 \\
\hline & Volatility (\%) & 7.91 & 8.10 & 7.68 \\
\hline & Skewness & 0.05 & 0.08 & -0.01 \\
\hline & Kurtosis & 3.66 & 2.85 & 4.63 \\
\hline & Minimum (\%) & -7.36 & -4.50 & -7.36 \\
\hline & Maximum (\%) & 9.40 & 7.30 & 9.40 \\
\hline \multirow[t]{6}{*}{ Cash (level) } & Mean (\%) & 4.46 & 6.21 & 2.71 \\
\hline & Volatility (\%) & 0.77 & 0.60 & 0.57 \\
\hline & Skewness & 0.16 & 0.45 & -0.01 \\
\hline & Kurtosis & 2.70 & 3.07 & 1.44 \\
\hline & Minimum(\%) & 0.00 & 0.00 & 0.00 \\
\hline & Maximum(\%) & 0.01 & 0.01 & 0.01 \\
\hline
\end{tabular}




\section{Table III. Average Risk-Adjusted Performance}

Classified by country and investment horizon, this table shows the average risk-adjusted performance of periodic rebalancing with yearly, quarterly, and monthly trading intervals over the sample period from January 1982 to December 2011. Panel A reports the average annualized Sharpe ratios, Panel B the average annualized Sortino ratios, and Panel C the average Omega measures. All strategies are based on a $60 \%$ stocks and $40 \%$ bonds asset allocation. The no-trade region comprises $\pm 3 \%$ around the target weights. Transaction costs are quoted at 15 bps per roundtrip. 1,000 simulations with an average block length of 2 are performed. Repeated simulations reveal that the results are stable.

\begin{tabular}{|c|c|c|c|c|}
\hline \multicolumn{5}{|c|}{ Panel A: Average Annualized Sharpe Ratios } \\
\hline Period & Rebalancing Strategy & United States & United Kingdom & Germany \\
\hline 5 & Yearly Rebalancing & 0.580 & 0.354 & 0.354 \\
\hline 5 & Quarterly Rebalancing & 0.583 & 0.356 & 0.359 \\
\hline 5 & Monthly Rebalancing & 0.580 & 0.355 & 0.356 \\
\hline 7 & Yearly Rebalancing & 0.597 & 0.398 & 0.369 \\
\hline 7 & Quarterly Rebalancing & 0.598 & 0.399 & 0.372 \\
\hline 7 & Monthly Rebalancing & 0.594 & 0.398 & 0.368 \\
\hline 10 & Yearly Rebalancing & 0.579 & 0.389 & 0.355 \\
\hline 10 & Quarterly Rebalancing & 0.579 & 0.390 & 0.356 \\
\hline 10 & Monthly Rebalancing & 0.575 & 0.389 & 0.351 \\
\hline \multicolumn{5}{|c|}{ Panel B: Average Annualized Sortino Ratios } \\
\hline Period & Rebalancing Strategy & United States & United Kingdom & Germany \\
\hline 5 & Yearly Rebalancing & 2.008 & 1.927 & 1.227 \\
\hline 5 & Quarterly Rebalancing & 2.027 & 1.942 & 1.246 \\
\hline 5 & Monthly Rebalancing & 2.022 & 1.941 & 1.242 \\
\hline 7 & Yearly Rebalancing & 1.945 & 1.939 & 1.191 \\
\hline 7 & Quarterly Rebalancing & 1.956 & 1.949 & 1.201 \\
\hline 7 & Monthly Rebalancing & 1.949 & 1.946 & 1.193 \\
\hline 10 & Yearly Rebalancing & 1.831 & 1.816 & 1.116 \\
\hline 10 & Quarterly Rebalancing & 1.836 & 1.821 & 1.122 \\
\hline 10 & Monthly Rebalancing & 1.828 & 1.817 & 1.112 \\
\hline \multicolumn{5}{|c|}{ Panel C: Average Omega Measures } \\
\hline Period & Rebalancing Strategy & United States & United Kingdom & Germany \\
\hline 5 & Yearly Rebalancing & 1.380 & 1.328 & 0.843 \\
\hline 5 & Quarterly Rebalancing & 1.393 & 1.340 & 0.856 \\
\hline 5 & Monthly Rebalancing & 1.390 & 1.339 & 0.853 \\
\hline 7 & Yearly Rebalancing & 1.334 & 1.331 & 0.815 \\
\hline 7 & Quarterly Rebalancing & 1.340 & 1.338 & 0.822 \\
\hline 7 & Monthly Rebalancing & 1.334 & 1.336 & 0.817 \\
\hline 10 & Yearly Rebalancing & 1.240 & 1.234 & 0.760 \\
\hline 10 & Quarterly Rebalancing & 1.243 & 1.237 & 0.764 \\
\hline 10 & Monthly Rebalancing & 1.237 & 1.234 & 0.758 \\
\hline
\end{tabular}




\section{Table IV. Confidence Intervals: Periodic Rebalancing}

Classified by country and investment horizon, this table shows the confidence intervals of the difference time series of periodic rebalancing of the average Sharpe ratio (Panel A), of the average Omega measure (Panel B), and of the average Sortino ratio (Panel C). The sample period ranges from January 1982 to December 2011. All strategies are based on a $60 \%$ stocks and $40 \%$ bonds asset allocation with a threshold of $0 \%$. Transaction costs are quoted at 15 bps per roundtrip. $\mathrm{Y}$ denotes yearly periodic rebalancing, Q quarterly periodic rebalancing, and M monthly periodic rebalancing. For example, M-Q denotes the difference time series of 'Monthly periodic rebalancing minus quarterly periodic rebalancing'. For each two strategies that are compared, the lower and upper boundary of the confidence interval is calculated. 1,000 simulations with an average block length of 2 are performed. Repeated simulations reveal that the results are stable. *, **, and *** represent statistical significance at the $10 \%, 5 \%$, and $1 \%$ level, respectively. If there is no statistical significance, the corresponding $10 \%$ quintiles are reported.

\begin{tabular}{|c|c|c|c|c|c|c|c|}
\hline \multicolumn{8}{|c|}{ Panel A: Average Annualized Sharpe Ratio of Periodic Rebalancing } \\
\hline Period & Strategies & \multicolumn{2}{|c|}{ United States } & \multicolumn{2}{|c|}{ United Kingdom } & \multicolumn{2}{|c|}{ Germany } \\
\hline 5 & M-Q & -0.0059 & $-0.0004 * * *$ & -0.0025 & 0.0002 & -0.0058 & $0.0000 * *$ \\
\hline 5 & $\mathrm{M}-\mathrm{Y}$ & -0.0042 & 0.0038 & -0.0016 & 0.0044 & -0.0024 & 0.0074 \\
\hline 5 & $\mathrm{Q}-\mathrm{Y}$ & -0.0005 & 0.0065 & 0.0000 & $0.0053 *$ & 0.0010 & $0.0101 *$ \\
\hline 7 & M-Q & -0.0065 & $-0.0015 * * *$ & -0.0028 & $-0.0001 *$ & -0.0078 & $-0.0010 * * *$ \\
\hline 7 & $M-Y$ & -0.0062 & 0.0011 & -0.0027 & 0.0030 & -0.0060 & 0.0032 \\
\hline 7 & $\mathrm{Q}-\mathrm{Y}$ & -0.0019 & 0.0044 & -0.0009 & 0.0042 & -0.0013 & 0.0071 \\
\hline 10 & M-Q & -0.0066 & $-0.0020 * * *$ & -0.0029 & $-0.0003 * *$ & -0.0081 & $-0.0022 * * *$ \\
\hline 10 & $M-Y$ & -0.0072 & $-0.0005 *$ & -0.0035 & 0.0015 & -0.0077 & 0.0003 \\
\hline 10 & $\mathrm{Q}-\mathrm{Y}$ & -0.0028 & 0.0032 & -0.0016 & 0.0028 & -0.0026 & 0.0049 \\
\hline \multicolumn{8}{|c|}{ Panel B: Average Annualized Sortino Ratio of Periodic Rebalancing } \\
\hline Period & Strategies & \multicolumn{2}{|c|}{ United States } & \multicolumn{2}{|c|}{ United Kingdom } & \multicolumn{2}{|c|}{ Germany } \\
\hline 5 & M-Q & -0.0100 & 0.0008 & -0.0060 & 0.0028 & -0.0102 & 0.0014 \\
\hline 5 & $\mathrm{M}-\mathrm{Y}$ & 0.0000 & 0.0278 & 0.0018 & $0.0271 * *$ & 0.0015 & $0.0285 *$ \\
\hline 5 & $\mathrm{Q}-\mathrm{Y}$ & 0.0003 & $0.0389 * * *$ & 0.0011 & $0.0316 * * *$ & 0.0042 & $0.0338 * *$ \\
\hline 7 & M-Q & -0.0126 & $-0.0014 * *$ & -0.0073 & 0.0009 & -0.0141 & $-0.0020 * *$ \\
\hline 7 & $\mathrm{M}-\mathrm{Y}$ & -0.0086 & 0.0144 & -0.0031 & 0.0168 & -0.0086 & 0.0147 \\
\hline 7 & Q-Y & 0.0000 & 0.0206 & 0.0010 & $0.0186 *$ & 0.0004 & $0.0221 *$ \\
\hline 10 & M-Q & -0.0149 & $-0.0015 * * *$ & -0.0068 & $-0.0003 *$ & -0.0169 & $-0.0034 * * *$ \\
\hline 10 & $\mathrm{M}-\mathrm{Y}$ & -0.0120 & 0.0076 & -0.0067 & 0.0094 & -0.0137 & 0.0055 \\
\hline 10 & $\mathrm{Q}-\mathrm{Y}$ & -0.0031 & 0.0152 & -0.0022 & 0.0124 & -0.0032 & 0.0148 \\
\hline \multicolumn{8}{|c|}{ Panel C: Average Omega Measure of Periodic Rebalancing } \\
\hline Period & Strategies & \multicolumn{2}{|c|}{ United States } & \multicolumn{2}{|c|}{ United Kingdom } & \multicolumn{2}{|c|}{ Germany } \\
\hline 5 & $\mathrm{M}-\mathrm{Q}$ & 0.0194 & -0.0068 & 0.0164 & -0.0037 & 0.0205 & -0.0057 \\
\hline 5 & $M-Y$ & 0.0006 & $0.0194 *$ & 0.0022 & $0.0201 * *$ & 0.0006 & $0.0215 * *$ \\
\hline 5 & $\mathrm{Q}-\mathrm{Y}$ & 0.0012 & $0.0274 * * *$ & 0.0013 & $0.0224 * * *$ & 0.0004 & $0.0253 * * *$ \\
\hline 7 & M-Q & -0.0108 & $-0.0011 * * *$ & -0.0047 & 0.0008 & -0.0085 & $-0.0010 * *$ \\
\hline 7 & $\mathrm{M}-\mathrm{Y}$ & -0.0070 & 0.0079 & -0.0016 & 0.0114 & -0.0048 & 0.0100 \\
\hline 7 & $\mathrm{Q}-\mathrm{Y}$ & -0.0002 & 0.0130 & 0.0012 & $0.0126 *$ & 0.0007 & $0.0142 *$ \\
\hline 10 & M-Q & -0.0110 & $-0.0027 * * *$ & -0.0055 & $-0.0004 * *$ & -0.0103 & $-0.0021 * * *$ \\
\hline 10 & $\mathrm{M}-\mathrm{Y}$ & -0.0095 & 0.0029 & -0.0052 & 0.0050 & -0.0082 & 0.0040 \\
\hline 10 & Q-Y & -0.0025 & 0.0086 & -0.0018 & 0.0074 & -0.0018 & 0.0097 \\
\hline
\end{tabular}




\section{Table V. Confidence Intervals: Threshold Rebalancing}

Classified by country and investment horizon, this table shows the confidence intervals of the difference time series of threshold rebalancing of the average Sharpe ratio (Panel A), of the average Omega measure (Panel B), and of the average Sortino ratio (Panel C). The sample period ranges from January 1982 to December 2011. All strategies are based on a $60 \%$ stocks and $40 \%$ bonds asset allocation with a threshold of $3 \%$. Transaction costs are quoted at 15 bps per roundtrip. Y denotes yearly threshold rebalancing, Q quarterly threshold rebalancing, and $\mathrm{M}$ monthly threshold rebalancing. For example, M-Q denotes the difference time series of 'Monthly threshold rebalancing minus quarterly threshold rebalancing'. For each two strategies that are compared, the lower and upper boundary of the confidence interval is calculated. 1,000 simulations with an average block length of 2 are performed. Repeated simulations reveal that the results are stable. *, **, and *** represent statistical significance at the $10 \%, 5 \%$, and $1 \%$ level, respectively. If there is no statistical significance, the corresponding $10 \%$ quintiles are reported.

\begin{tabular}{|c|c|c|c|c|c|c|c|}
\hline \multicolumn{8}{|c|}{ Panel A: Average Annualized Sharpe Ratio of Threshold Rebalancing } \\
\hline Period & Strategies & \multicolumn{2}{|c|}{ United States } & \multicolumn{2}{|c|}{ United Kingdom } & \multicolumn{2}{|c|}{ Germany } \\
\hline 5 & M-Q & -0.0032 & 0.0014 & -0.0021 & 0.0015 & -0.0043 & 0.0012 \\
\hline 5 & $M-Y$ & -0.0022 & 0.0058 & -0.0007 & 0.0051 & -0.0013 & 0.0083 \\
\hline 5 & $\mathrm{Q}-\mathrm{Y}$ & -0.0011 & 0.0065 & -0.0002 & 0.0053 & 0.0000 & $0.0107 * *$ \\
\hline 7 & M-Q & -0.0036 & 0.0006 & -0.0024 & 0.0012 & -0.0049 & $-0.0002 *$ \\
\hline 7 & $\mathrm{M}-\mathrm{Y}$ & -0.0038 & 0.0034 & -0.0021 & 0.0038 & -0.0045 & 0.0046 \\
\hline 7 & $\mathrm{Q}-\mathrm{Y}$ & -0.0021 & 0.0045 & -0.0011 & 0.0041 & -0.0016 & 0.0069 \\
\hline 10 & M-Q & -0.0038 & $0.0000 *$ & -0.0025 & 0.0005 & -0.0066 & $-0.0003 * * *$ \\
\hline 10 & $M-Y$ & -0.0049 & 0.0018 & -0.0028 & 0.0024 & -0.0061 & 0.0018 \\
\hline 10 & $\mathrm{Q}-\mathrm{Y}$ & -0.0027 & 0.0035 & -0.0018 & 0.0031 & -0.0026 & 0.0050 \\
\hline \multicolumn{8}{|c|}{ Panel B: Average Annualized Sortino Ratio of Threshold Rebalancing } \\
\hline Period & Strategies & \multicolumn{2}{|c|}{ United States } & \multicolumn{2}{|c|}{ United Kingdom } & \multicolumn{2}{|c|}{ Germany } \\
\hline 5 & M-Q & -0.0075 & 0.0083 & -0.0055 & 0.0071 & -0.0092 & 0.0053 \\
\hline 5 & $M-Y$ & 0.0004 & $0.0286 *$ & 0.0006 & $0.0254 * *$ & 0.0011 & $0.0279 *$ \\
\hline 5 & $\mathrm{Q}-\mathrm{Y}$ & 0.0010 & $0.0277 *$ & 0.0002 & $0.0248 * *$ & 0.0016 & $0.0313 * *$ \\
\hline 7 & M-Q & -0.0083 & 0.0055 & -0.0070 & 0.0052 & -0.0106 & 0.0017 \\
\hline 7 & $M-Y$ & -0.0054 & 0.0180 & -0.0049 & 0.0161 & -0.0076 & 0.0167 \\
\hline 7 & $\mathrm{Q}-\mathrm{Y}$ & -0.0030 & 0.0192 & -0.0022 & 0.0163 & -0.0021 & 0.0202 \\
\hline 10 & M-Q & -0.0093 & 0.0029 & -0.0079 & $-0.0008 * *$ & -0.0118 & 0.0029 \\
\hline 10 & $M-Y$ & -0.0089 & 0.0112 & -0.0073 & 0.0096 & -0.0120 & 0.0075 \\
\hline 10 & $\mathrm{Q}-\mathrm{Y}$ & -0.0056 & 0.0138 & -0.0053 & 0.0115 & -0.0049 & 0.0135 \\
\hline \multicolumn{8}{|c|}{ Panel C: Average Omega Measure of Threshold Rebalancing } \\
\hline Period & Strategies & \multicolumn{2}{|c|}{ United States } & \multicolumn{2}{|c|}{ United Kingdom } & \multicolumn{2}{|c|}{ Germany } \\
\hline 5 & M-Q & -0.0053 & 0.0053 & -0.0036 & 0.0051 & -0.0053 & 0.0033 \\
\hline 5 & M-Y & 0.0002 & $0.0187 *$ & 0.0003 & $0.0180 * *$ & 0.0007 & $0.0187 *$ \\
\hline 5 & $\mathrm{Q}-\mathrm{Y}$ & 0.0009 & $0.0183 *$ & 0.0005 & $0.0172 * *$ & 0.0017 & $0.0201 * *$ \\
\hline 7 & M-Q & -0.0063 & 0.0028 & -0.0047 & 0.0033 & -0.0065 & 0.0009 \\
\hline 7 & M-Y & -0.0048 & 0.0102 & -0.0033 & 0.0105 & -0.0049 & 0.0106 \\
\hline 7 & $\mathrm{Q}-\mathrm{Y}$ & -0.0027 & 0.0117 & -0.0016 & 0.0107 & -0.0013 & 0.0126 \\
\hline 10 & M-Q & -0.0064 & 0.0010 & -0.0047 & 0.0014 & -0.0078 & $-0.0006 * *$ \\
\hline 10 & $M-Y$ & -0.0069 & 0.0058 & -0.0055 & 0.0053 & -0.0076 & 0.0048 \\
\hline 10 & $\mathrm{Q}-\mathrm{Y}$ & -0.0039 & 0.0079 & -0.0036 & 0.0067 & -0.0031 & 0.0083 \\
\hline
\end{tabular}




\section{Table VI. Confidence Intervals: Range Rebalancing}

Classified by investment horizon, this table shows the confidence intervals of the difference time series of range rebalancing of the average Sharpe ratio (Panel A), of the average Omega measure (Panel B), and of the average Sortino ratio (Panel C). The sample period ranges from January 1982 to December 2011. All strategies are based on a $60 \%$ stocks and $40 \%$ bonds asset allocation with a threshold of $3 \%$. Transaction costs are quoted at 15 bps per roundtrip. Y denotes yearly range rebalancing, Q quarterly range rebalancing, and $\mathrm{M}$ monthly range rebalancing. For example, M-Q denotes the difference time series of 'Monthly range rebalancing minus quarterly range rebalancing'. For each two strategies that are compared, the lower and upper boundary of the confidence interval is calculated. 1,000 simulations with an average block length of 2 are performed. Repeated simulations reveal that the results are stable. *,**, and *** represent statistical significance at the $10 \%$, 5\%, and $1 \%$ level, respectively. If there is no statistical significance, the corresponding $10 \%$ quintiles are reported.

\begin{tabular}{|c|c|c|c|c|c|c|c|}
\hline \multicolumn{8}{|c|}{ Panel A: Average Annualized Sharpe Ratio of Range Rebalancing } \\
\hline Period & Strategies & \multicolumn{2}{|c|}{ United States } & \multicolumn{2}{|c|}{ United Kingdom } & \multicolumn{2}{|c|}{ Germany } \\
\hline 5 & $M-Q$ & -0.0002 & 0.0021 & 0.0000 & $0.0018 *$ & 0.0002 & $0.0034 *$ \\
\hline 5 & $M-Y$ & 0.0018 & $0.0099 * * *$ & 0.0014 & $0.0076 * * *$ & 0.0038 & $0.0159 * * *$ \\
\hline 5 & $\mathrm{Q}-\mathrm{Y}$ & 0.0017 & $0.0084 * * *$ & 0.0009 & $0.0065 * * *$ & 0.0026 & $0.0133 * * *$ \\
\hline 7 & M-Q & -0.0008 & 0.0013 & -0.0004 & 0.0013 & -0.0007 & 0.0022 \\
\hline 7 & $M-Y$ & 0.0007 & $0.0071 * *$ & 0.0005 & $0.0056 * *$ & 0.0006 & $0.0125 * * *$ \\
\hline 7 & $\mathrm{Q}-\mathrm{Y}$ & 0.0000 & $0.0069 * * *$ & 0.0000 & $0.0059 * * *$ & 0.0005 & $0.0110 * * *$ \\
\hline 10 & M-Q & -0.0012 & 0.0008 & -0.0008 & 0.0007 & -0.0012 & 0.0013 \\
\hline 10 & $M-Y$ & -0.0001 & 0.0049 & -0.0004 & 0.0036 & 0.0001 & $0.0081 * *$ \\
\hline 10 & $\mathrm{Q}-\mathrm{Y}$ & 0.0001 & $0.0051 * *$ & 0.0000 & $0.0034 *$ & 0.0005 & $0.0076 * *$ \\
\hline \multicolumn{8}{|c|}{ Panel B: Average Annualized Sortino Ratio of Range Rebalancing } \\
\hline Period & Strategies & \multicolumn{2}{|c|}{ United States } & \multicolumn{2}{|c|}{ United Kingdom } & \multicolumn{2}{|c|}{ Germany } \\
\hline 5 & M-Q & 0.0013 & $0.0105 * *$ & 0.0010 & $0.0099 * * *$ & 0.0012 & $0.0111 * *$ \\
\hline 5 & $M-Y$ & 0.0139 & $0.0444 * * *$ & 0.0092 & $0.0359 * * *$ & 0.0135 & $0.0472 * * *$ \\
\hline 5 & $\mathrm{Q}-\mathrm{Y}$ & 0.0102 & $0.0380 * * *$ & 0.0062 & $0.0287 * * *$ & 0.0091 & $0.0375 * * *$ \\
\hline 7 & $M-Q$ & -0.0001 & 0.0068 & 0.0000 & $0.0061 *$ & -0.0006 & 0.0070 \\
\hline 7 & $M-Y$ & 0.0058 & $0.0358 * * *$ & 0.0024 & $0.0297 * * *$ & 0.0041 & $0.0369 * * *$ \\
\hline 7 & $\mathrm{Q}-\mathrm{Y}$ & 0.0054 & $0.0296 * * *$ & 0.0021 & $0.0243 * * *$ & 0.0037 & $0.0303 * * *$ \\
\hline 10 & M-Q & -0.0014 & 0.0045 & -0.0015 & 0.0034 & -0.0022 & 0.0041 \\
\hline 10 & $\mathrm{M}-\mathrm{Y}$ & 0.0016 & $0.0264 * * *$ & 0.0007 & $0.0170 * *$ & 0.0002 & $0.0264 * * *$ \\
\hline 10 & $\mathrm{Q}-\mathrm{Y}$ & 0.0025 & $0.0232 * * *$ & 0.0010 & $0.0148 * *$ & 0.0014 & $0.0251 * * *$ \\
\hline \multicolumn{8}{|c|}{ Panel C: Average Omega Measure of Range Rebalancing } \\
\hline Period & Strategies & \multicolumn{2}{|c|}{ United States } & \multicolumn{2}{|c|}{ United Kingdom } & \multicolumn{2}{|c|}{ Germany } \\
\hline 5 & M-Q & 0.0007 & $0.0070 * *$ & 0.0006 & $0.0071 * * *$ & 0.0010 & $0.0073 * *$ \\
\hline 5 & $M-Y$ & 0.0090 & $0.0323 * * *$ & 0.0065 & $0.0257 * * *$ & 0.0083 & $0.0317 * * *$ \\
\hline 5 & $\mathrm{Q}-\mathrm{Y}$ & 0.0071 & $0.0268 * * *$ & 0.0046 & $0.0200 * * *$ & 0.0060 & $0.0252 * * *$ \\
\hline 7 & M-Q & -0.0007 & 0.0040 & -0.0001 & 0.0042 & -0.0003 & 0.0045 \\
\hline 7 & M-Y & 0.0033 & $0.0218 * * *$ & 0.0010 & $0.0205 * * *$ & 0.0024 & $0.0235 * * *$ \\
\hline 7 & $\mathrm{Q}-\mathrm{Y}$ & 0.0033 & $0.0194 * * *$ & 0.0009 & $0.0164 * * *$ & 0.0025 & $0.0205 * * *$ \\
\hline 10 & M-Q & -0.0011 & 0.0027 & -0.0013 & 0.0020 & -0.0013 & 0.0026 \\
\hline 10 & M-Y & 0.0008 & $0.0169 * * *$ & 0.0004 & $0.0096 *$ & 0.0001 & $0.0172 * * *$ \\
\hline 10 & $\mathrm{Q}-\mathrm{Y}$ & 0.0012 & $0.0146 * * *$ & 0.0003 & $0.0086 *$ & 0.0009 & $0.0157 * * *$ \\
\hline
\end{tabular}




\section{Table VII. Confidence Intervals: Optimal Rebalancing}

Classified by investment horizon, this table shows the confidence intervals of the difference time series of the average annualized Sharpe ratio between quarterly periodic rebalancing, threshold rebalancing, and monthly range rebalancing. The sample period ranges from January 1982 to December 2011. All strategies are based on a $60 \%$ stocks and $40 \%$ bonds asset allocation. The no-trade region comprises $\pm 3 \%$ around the target weights. Transaction costs are quoted at 15 bps per roundtrip. For example, Periodic-Range denotes the difference time series of 'Quarterly periodic rebalancing minus monthly range rebalancing'. For each two strategies that are compared, the lower and upper boundary of the confidence interval is calculated. 1,000 simulations with an average block length of 2 are performed. Repeated simulations reveal that the results are stable. ${ }^{*}, * *$, and $* * *$ represent statistical significance at the $10 \%, 5 \%$, and $1 \%$ level, respectively. If there is no statistical significance, the corresponding $10 \%$ quintiles are reported.

\begin{tabular}{|c|c|c|c|c|c|c|c|}
\hline \multicolumn{8}{|c|}{ Panel A: Average Annualized Sharpe Ratio } \\
\hline Period & Strategies & \multicolumn{2}{|c|}{ United States } & \multicolumn{2}{|c|}{ United Kingdom } & \multicolumn{2}{|c|}{ Germany } \\
\hline 5 & Periodic - Threshold & -0.0004 & 0.0036 & -0.0006 & 0.0025 & -0.0007 & 0.0029 \\
\hline 5 & Periodic - Range & 0.0024 & $0.0102 * * *$ & 0.0010 & $0.0069 * * *$ & 0.0010 & $0.0088 * * *$ \\
\hline 5 & Threshold - Range & 0.0007 & $0.0079 * * *$ & 0.0001 & $0.0058 * * *$ & 0.0003 & $0.0079 * * *$ \\
\hline 7 & Periodic - Threshold & -0.0007 & 0.0029 & -0.0005 & 0.0024 & -0.0008 & 0.0024 \\
\hline 7 & Periodic - Range & 0.0010 & $0.0082 * * *$ & 0.0008 & $0.0068 * * *$ & 0.0005 & $0.0076 * * *$ \\
\hline 7 & Threshold - Range & 0.0002 & $0.0065 * * *$ & 0.0006 & $0.0047 * *$ & 0.0003 & $0.0059 * *$ \\
\hline 10 & Periodic - Threshold & -0.0008 & 0.0021 & -0.0007 & 0.0018 & -0.0008 & 0.0019 \\
\hline 10 & Periodic - Range & 0.0000 & $0.0063 * * *$ & 0.0004 & $0.0045 * *$ & 0.0006 & $0.0053 * *$ \\
\hline 10 & Threshold - Range & 0.0003 & $0.0048 * *$ & 0.0001 & $0.0037 * *$ & 0.0002 & $0.0047 * *$ \\
\hline \multicolumn{8}{|c|}{ Panel B: Average Annualized Sortino Ratio } \\
\hline Period & Strategies & \multicolumn{2}{|c|}{ United States } & \multicolumn{2}{|c|}{ United Kingdom } & \multicolumn{2}{|c|}{ Germany } \\
\hline 5 & Periodic - Threshold & 0.0026 & $0.0197 * *$ & 0.0014 & $0.0191 * * *$ & 0.0008 & $0.0109 *$ \\
\hline 5 & Periodic - Range & 0.0179 & $0.0472 * * *$ & 0.0166 & $0.0414 * * *$ & 0.0088 & $0.0303 * * *$ \\
\hline 5 & Threshold - Range & 0.0078 & $0.0349 * * *$ & 0.0071 & $0.0292 * * *$ & 0.0035 & $0.0240 * * *$ \\
\hline 7 & Periodic - Threshold & 0.0010 & $0.0154 * *$ & 0.0000 & $0.0174 * * *$ & 0.0003 & $0.0090 *$ \\
\hline 7 & Periodic - Range & 0.0119 & $0.0360 * * *$ & 0.0113 & $0.0338 * * *$ & 0.0058 & $0.0253 * * *$ \\
\hline 7 & Threshold - Range & 0.0049 & $0.0268 * * *$ & 0.0051 & $0.0240 * * *$ & 0.0018 & $0.0211 * * *$ \\
\hline 10 & Periodic - Threshold & 0.0004 & $0.0114 * *$ & 0.0006 & $0.0113 * *$ & 0.0002 & $0.0070 *$ \\
\hline 10 & Periodic - Range & 0.0077 & $0.0263 * * *$ & 0.0062 & $0.0252 * * *$ & 0.0042 & $0.0192 * * *$ \\
\hline 10 & Threshold - Range & 0.0029 & $0.0211 * * *$ & 0.0019 & $0.0177 * * *$ & 0.0008 & $0.0162 * * *$ \\
\hline \multicolumn{8}{|c|}{ Panel C: Average Omega Measure } \\
\hline Period & Strategies & \multicolumn{2}{|c|}{ United States } & \multicolumn{2}{|c|}{ United Kingdom } & \multicolumn{2}{|c|}{ Germany } \\
\hline 5 & Periodic - Threshold & 0.0010 & $0.0159 * * *$ & 0.0020 & $0.0146 * * *$ & 0.0005 & $0.0080 * *$ \\
\hline 5 & Periodic - Range & 0.0127 & $0.0346 * * *$ & 0.0127 & $0.0315 * * *$ & 0.0067 & $0.0208 * * *$ \\
\hline 5 & Threshold - Range & 0.0055 & $0.0249 * * *$ & 0.0057 & $0.0213 * * *$ & 0.0033 & $0.0160 * * *$ \\
\hline 7 & Periodic - Threshold & 0.0012 & $0.0101 * *$ & 0.0012 & $0.0122 * * *$ & 0.0005 & $0.0065 * *$ \\
\hline 7 & Periodic - Range & 0.0088 & $0.0244 * * *$ & 0.0096 & $0.0250 * * *$ & 0.0051 & $0.0174 * * *$ \\
\hline 7 & Threshold - Range & 0.0036 & $0.0181 * * *$ & 0.0046 & $0.0172 * * *$ & 0.0021 & $0.0142 * * *$ \\
\hline 10 & Periodic - Threshold & 0.0004 & $0.0072 * *$ & 0.0003 & $0.0085 * * *$ & 0.0003 & $0.0051 * *$ \\
\hline 10 & Periodic - Range & 0.0051 & $0.0171 * * *$ & 0.0056 & $0.0177 * * *$ & 0.0037 & $0.0136 * * *$ \\
\hline 10 & Threshold - Range & 0.0022 & $0.0138 * * *$ & 0.0021 & $0.0122 * * *$ & 0.0014 & $0.0109 * * *$ \\
\hline
\end{tabular}

